\title{
LA CONQUETE DE L'ESPACE VARIATION SUR UN ART DE LA REENCONTRE : LE GESTE BAROQUE
}

\author{
Yannick Butel \\ Professeur des Universités en Arts de la scène \\ Aix-Marseille Université, laboratoire d'études en sciences des arts \\ yannick.butel@sfr.fr
}

Résumé

Si cet article est critique, il l'est à l'endroit des finalités et des visées des pratiques théâtrales qui, sous la contrainte et la conjonction d'un ensemble idéologico-économique, sont finalement, à leur insu ou pas, au service de l'intérêt et du souci du « faire communauté ». Et ce parce que l'organisation structurelle du théâtre (aussi bien les constituants théâtraux que l'industrie du spectacle) participe d'un programme de « fabrique du commun » où est préférée la notion de public (et donc de peuple) à la notion de sujet (et donc de singularité quelconque). À cette organisation tactique du phénomène théâtral, les pratiques en espaces ouverts seront alors considérées comme une alternative incarnant un modèle de "biopolitique" qui a pour objet de libérer la vie, de la soustraire au dressage institutionnel tel qu'il a pu être commenté dans La Volonté de savoir par Michel Foucault lorsqu'il analyse le « bio-pouvoir ».

Mots clé

Colonisation de la Pensée. Pratiques Urbaines. Espace Public. Industrie Culturelle. Théâtre.
Resumo

Se esse artigo tem um ponto de vista crítico, é porque ele foca nas finalidades e objetivos das práticas teatrais que, sob uma conjuntura ideológico-econômica, cientes ou não disso, se encontram a serviço do interesse e da preocupação do "fazer comunidade". Isso se dá em razão da organização estrutural do teatro (tanto os elementos teatrais quando a indústria do espetáculo) que faz parte de uma ideia de "fábrica do comum", onde é preferível a noção de público (e consequentemente de povo) à noção de indivíduo (ou seja, de singularidade banal, anônima). Em vista de tal organização estratégica do fenômeno teatral, as práticas em espaços abertos podem, então, ser consideradas como uma alternativa que incorpora o modelo de "biopolítica", cujo objetivo é o de libertar a vida, de retirá-la do adestramento institucional comentado por Michel Foucault em "A vontade do saber", quando ele analisa o "biopoder".

Palavras-chave

Colonização do Pensamento. Práticas Urbanas. Espaço Público. Indústria Cultural. Teatro. 
L'espace est formé par un ensemble indissociable à la fois solidaire et contradictoire, de systèmes d'objets et de systèmes d'actions, cadre unique dans lequel se fait l'histoire. ${ }^{1}$

Une immense part de la population est aujourd'hui privée de toute expérience esthétique, entièrement soumise qu'elle est au conditionnement esthétique. $^{2}$

la production capitaliste a unifié l'espace, qui n'est plus limité par des sociétés extérieures. Cette unification est en même temps un processus extensif et intensif de banalisation. ${ }^{3}$

Le caractère de l'ensemble du monde est de toute éternité celui du chaos, en raison [...] de l'absence d'ordre. ${ }^{4}$

Dans l'une des plus importantes pièces de Bertolt Brecht Mère courage et ses enfants, écrite en 1939 - Brecht est alors en exil aux USA - puis mise en scène en 1949 avant que Peter Palitzsch n'en réalise un film en 1960 avec les comédiens du Berliner Ensemble, la scène d'exposition présente des gravures de guerre : scènes de bataille, arbre aux pendus et charniers. Apparaît à la suite la carriole d'Anna Fierling, tirée par ses enfants Eilif et Schweizerkas, le long d'une route bordée d'arbres morts, dans un paysage désert...

Dans Place des héros, ultime pièce de Thomas Bernhard (Heldenplatz,1988) mise en scène au Festival d'Avignon en 2016 par Krys-

1 Santos, Milton. La Nature de l'espace, technique et temps, raison et émotion, coll. In: Géographie en liberté. Paris: L'Harmattan, 1997. p. 44.

2 Stiegler, Bernard. De la misère symbolique. Paris: Galilée, 2004, p. 20-21.

3 Debord, Guy. La Société du spectacle. Paris: Gallimard, 1992, p. 129.

4 Nietzsche, Friedrich. Le Gai savoir: aphorisme 109. Trad. P. Klossowski. Paris: Gallimard, 1982. p. 138. tian Lupa (initialement créée en 2015 au Théâtre National de Lituanie à Vilnius), Madame Schuster, dans un appartement bourgeois viennois, se tient derrière une immense fenêtre irradiée de lumière vive qui donne sur la place où cinquante ans plus tôt, en 1938, à l'occasion de l'Anschluss entre l'Allemagne et l'Autriche, le chancelier Hitler était acclamé par la foule. Le professeur Schuster vient de se défenestrer. Les clameurs de la foule sont audibles.

Dans Soubresaut, dernière création de François Tanguy au Théâtre National de Bretagne dans le cadre du festival « Mettre en scène », reprise en décembre 2016 à la Fonderie le Mans, mêlé aux poèmes et autres textes de Celan, Dante, Kafka, Coleridge... qui sont dits, le bruit de bombardements aériens durant la Seconde Guerre mondiale s'entend au lointain. Le volume des bombardements est plus ou moins fort et menace l'harmonie musicale et littéraire comme le rythme des phrases ciselées...

\section{De la « communauté sensible»}

Au prétexte de ces trois épisodes, il ne s'agit pas d'analyser ceux-ci pour en extraire quelques commentaires propres à satisfaire l'interprétation. Notre propos n'est pas guidé par le souci de l'exégèse qui tendrait à éclairer la signification de ces créations. Convoquant ces trois mises en scène, il s'agit plutôt de revenir sur l'enjeu qu'est l'espace dans le processus théâtral et de proposer une réflexion sur celui-ci, eu égard la citation de Milton Santos que nous avons placée en exergue de cet article. Soit, et précisons-le, de montrer en quoi la complexité de la construction des espaces dans ces processus théâtraux, et les constituants qui y participent, ont un lien étroit avec 
l'écriture de l'Histoire, au sens où ils y contribuent. Proposition qui, sauf à vider l'Histoire de son contenu, induit de fait la question du rapport qu'entretiennent la politique et l'esthétique ${ }^{5}$.

A priori, notre approche ne relève d'aucune originalité puisque depuis longtemps déjà, " Théâtre, Histoire, Esthétique et Politique » sont dans un rapport consubtantiel.

Dès lors, pourquoi y revenir?

II est nécessaire d'y revenir parce que les études sur la scénographie ${ }^{6}$, comme celles qui concernent les imbrications entre le Théâtre, l'Histoire, l'Esthétique et le Politique, n'ont de cesse de reconduire un invariant structurel qui constitue l'espace théâtral comme un « espace

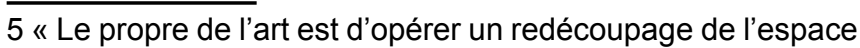
matériel et sympbolique. Et c'est par là que l'art touche au politique. La politique, en effet, ce n'est pas l'exercice du pouvoir et la lutte pour le pouvoir. C'est la configuration d'un espace spécifique, le découpage d'une sphère particulière d'expérience, d'objets posés comme communs et relevant d'une décision commune... ", Jacques Rancière, Malaise dans l'esthétique, Paris, Galilée, 2004, p. 37. Insistons sur le détail de cette situation en soulignant que cette définition connecte l'esthétique et le politique à l'espace. D'où notre intérêt de faire cette citation.

6 On doit ainsi distinguer les études sur la scénographie, de ceux qui sont scénographes et ont un rapport critique à l'espace. Notamment, les scénographes des années 60-70, au sein de l'Arte Povera, comme Jannis Kounellis, qui expriment des doutes quant à l'espace et au phénomène théâtral, leur préférant l'action artistique en milieu urbain. Je cite : « D'abord je ne vais pas au théâtre. Pourquoi aller au théâtre quand tout est prévu ou prévisible, quand l'ancien prévaut encore tellement sur le nouveau ? Un artiste fuit cela, mais s'il décide d'intervenir, il ne peut qu'adopter une attitude polémique envers ce qu'est le théâtre, envers ce qui masque l'emprise d'idées sclérosées et cherche en fait à éviter tout changement [...] mes matériaux prétendent à un espace propre, et en même temps créent un espace complexe qui tend moins à faire oublier la fiction du lieu théâtral qu'à la remettre en question, à la provoquer, à révéler les contraintes qu'elle implique », explique Jannis Kounellis. In «Après la scénographie », entretien dirigé par Guido Boursier, Italo Moscati et Marisa Rusconi, in Sipario, $n^{\circ} 276$, avril 1969 . On pourrait ajouter les expériences du Festival de Nancy, celle du Living theater à Avignon, celle du Magic Circus au festival Sigma de Bordeaux, de JeanJacques Lebel, des happenings du Palais des Merveilles, du travail urbain du Théâtre de l'unité de Jacques Livchine, etc. qui sont hors les murs et vécues comme un théâtre d'agitation urbaine. à part », une entité « séparée »7 comme le relevait de manière critique Guy Debord. Comprenons par là, un lieu où le changement d'échelle (ce que l'on appelle la fiction, voire l'art) permettrait d'obtenir un effet de visibilité, de lisibilité et de justesse inégalable, propre, en définitive, à la technique et à la pratique artistiques. Cet invariant, pour autant qu'il constitue le théâtre comme un art à part entière, le maintient paradoxalement à la marge de la réalité du monde qui semble, elle, indépassablement réglée par un principe de myopie puisque ce qui est perçu au théâtre n'est perceptible nulle part ailleurs.

Par divers processus d'ajustements rigoureux, d'augmentations précises, de mise en relief d'une acuité incroyable, de mise en avant judicieuse... le théâtre est donc le lieu où l'on voit/d'où l'on voit. Jusqu'au dispositif de mise en visibilité qui se préoccupe de la place du spectateur qui doit voir, tout est ainsi pensé par rapport à cet enjeu optique qui abrite un enjeu sensible.

Et d'entendre les commentaires politiques ou ceux de la communauté artistique, relayer cette pertinence fabuleuse, cette supériorité incroyable où l'on finit par faire d'une image comme d'un son poétique un original ; quand la réalité du monde - cet espace moisi - n'est plus appréhendée que sous la forme d'une copie brouillonne ${ }^{8}$. 7 « le spectacle se présente à la fois comme la société même,
comme une partie de la société, et comme instrument d'unifi-
cation. En tant que partie de la société, il est expressément le
secteur qui concentre tout regard et toute conscience. Du fait
même que ce secteur est séparé, il est le lieu du regard abusé
et de la fausse conscience ; et l'unification qu'il accomplit n'est
rien d'autre qu'un langage officiel de la séparation généralisée
». In: Debord, Guy. La Société... op. cit.,p. 4 .

8 Ce constat est l'objet de la critique de Debord. Citant Feuerbach : «Et sans doute notre temps... préfère l'image à la chose, la copie à l'original, la représentation à la réalité, l'apparence à l'être... Ce qui est sacré pour lui, ce n'est que l'illusion, mais ce qui est profane, c'est la vérité » (ibid., p. 132), 
«Commentaire politique » comme celui de Malraux sur l'art, par exemple, qui lors de son voyage au Brésil ${ }^{9}$, (qu'il nomme « le pays de l'espoir ») à Rio de Janeiro, puis à Brasilia (" cette Brasilia sur son plateau géant, c'est un peu l'Acropole sur son rocher... » écrit-il) avant de se rendre à la faculté de philosophie de São Paulo, n'a de cesse de commenter les classiques (Sophocle, Corneille, Shakespeare...), entre autres, et de ponctuer son voyage politique le 26 aout 1959 à São Paulo, par un discours où il pense le rôle de l'art comme révélateur de la dignité humaine.

«Commentaire esthétique » où les penseurs du théâtre théorisent le travail des dramaturges, tel Bernard Dort à propos de Bertolt Brecht :

son théâtre épique apparaît ainsi comme une entreprise de déconditionnement et de destruction des idéologies. C'est en portant à la scène les mythes de la vie quotidienne, notre façon de vivre au jour le jour l'Histoire, voire une certaine théâtralité de cette vie quotidienne (nos gestes et nos opinions...) que Brecht nous offre le moyen de prendre du champ par rapport à cette vie quotidienne et de nous en dégager. L'objet d'un tel théâtre, ce sont les fausses représentations que l'homme se fait de lui-même et de la société. (Dort, 1960, p. 199). ${ }^{10}$

\footnotetext{
l'analyse de Debord souligne cette confusion tout en relevant qu'il y a une intention et une volonté du pouvoir d'entretenir celle-ci :" Le spectacle, compris dans sa totalité, est à la fois le résultat et le projet du mode de production existant. II n'est pas un supplément au monde réel, sa décoration surajoutée. Il est le cœur de l'irréalisme de la société réelle. Sous toutes ses formes particulières, information ou propagande, publicité ou consommation directe de divertissements, le spectacle constitue le modèle présent de la vie socialement dominante ». Ibid., p. 5.

9 Sur cet épisode, consulter l'article d'Edson Rosa Da Silva, « André Malraux au Brésil : le rôle de l'art ». Article consulté sur : https://www.cairn.info/revue-de-litterature-comparee-2005-4page-443.htm\#no5. 19 décembre 2016.
}

10 Dort, Bernard. Lecture de Brecht. Paris: Seuil, 1960, p. 199.
II serait possible de multiplier ces paroles sur l'art, et notamment l'art du théâtre assigné à « être populaire » et au « service de ».

"Révéler, reconnaître, éveiller, démystifier, éduquer... » dans la perception que l'on a de l'utilité de l'art théâtral, il est évident que celui-ci est pris pour le miroir magique qui permet de voir ce qui était insoupçonnable. Qualité d'un art du vrai qui doit nous écarter " des fausses représentations " écrit Dort à propos du travail de Brecht ; et où l'art serait un « révélateur » pense et écrit Malraux.

Mais comment ne pas suspecter cette alliance du politique et du théoricien ? comment ne pas être intrigué par cet accord qui porte ici sur l'espace théâtral et sa finalité ? Comment ne pas s'inquiéter d'une telle convergence et collusion?

Il faut donner raison à Bernard Stiegler quand il écrit : " la question politique est essentiellement la question de la relation à l'autre dans un sentir ensemble [...] La politique est l'art de garantir une unité de la cité dans son désir d'avenir commun [...] Or un tel désir suppose un fonds esthétique commun. L'être-ensemble est celui d'un ensemble sensible. Une communauté politique est donc la communauté d'un sentir »11.

Et d'ajouter que dans cette perspective, les uns et les autres, voire parfois les créateurs, oeuvrent à la constitution d'une « communauté sensible " assemblée, in fine, autour d'une culture. C'est-à-dire, un ordre esthétique qui se confond avec un ordre politique dont la finalité est de contrôler l'espace via la culture qui s'y déploie.

Dès lors, et pour revenir au doute que nous exprimions et qui suscite cette analyse, la lé-

11 Stiegler, Bernard. De la misère symbolique, op.cit., p. 1819. 
gitimité du clivage entre les espaces (espaces fictions/espaces de la réalité) se justifie par l'impératif politique d'entretenir une dualité qui forme en définitive une unité qui est l'autre nom ou la variation de la « communauté sensible ». En maintenant l'art théâtral dans cette configuration, les œuvres du théâtre s'inscrivent donc dans un art du dévoilement où le « sentir » est le symptôme de la communauté ; " se sentir » s'obtenant sur le principe d'échangeabilité qui règle le commerce du spectateur avec l'objet artistique qu'il rencontre et que commente Jean-François Lyotard.

Au vrai, l'art théâtral est donc un art aléthique et un espace où le reflet, corrigé et augmenté que le théâtre offre de la société carencée, donne la consistance qui manquait à celle-ci. De là la confusion qui s'ensuit et dont nous faisions la critique : confondre la copie avec la réalité, se méprendre sur le rapport que l'original entretient avec la représentation, sous-estimer le modèle et lui préférer le modèle dérivé, préférer l'image du miroir aux traits qu'offre le monde, s'émouvoir à l'endroit de la fiction quand la réalité indiffère...

Soit, et pour le formuler de façon laconique, une manière de penser et légitimer l'espace théâtral (la schize que nous avons soulignée) en l'apparentant, dans son fonctionnement et ses fins, à celui du stade du miroir.

Mais qu'en serait-il de ce clivage si l'art du théâtre était affranchi de cette emprise idéologique ? Si la pratique du théâtre se déprenait de cet adossement systématique à la communauté sensible? Qu'en serait-il si les effets de la colonisation ${ }^{12}$ de la pensée sur le théâtre

12 Entendons par l'expression « colonisation de la pensée » une manière de nommer les invariants structurels de l'art théâtral : communauté assemblée, pratique artistique arraisonnée à la communication, espace de partage des sensations, jeu dialectique entre réalité/fiction, division de l'espace, etc. Ajou- étaient pris en compte, voire venaient à s'estomper ou disparaître ? Que serait une pratique du théâtre, un art, qui ne serait plus au service de la culture qui, rappelons-le, n'est représentative que d'un ordre esthético-politique?

A priori, l'hypothèse semble incongrue puisqu'elle vient se heurter à une réflexion sur le théâtre qui, déclinée au long des siècles, de La Poétique d'Aristote au Théâtre postdramatique de Lehmann- lequel marque le « désenchantement post-moderne » et le deuil de la communauté, n'est qu'une variation sur le thème récurrent du « partage du sensible » présent ou remis en cause.

Cela étant, risquons-nous à l'exploration de cette hypothèse.

\section{L'Écho et le point d'appui}

En convoquant les trois exemples de mises en scène, nous tenterons de penser cet enjeu qu'est l'espace, autrement. Revenons donc sur ceux-ci.

Chez Brecht, Mère Courage et ses enfants est construit d'emblée sur la projection de gravures qui, dans un rapport mimétique à la guerre de Trente ans, fonctionne tels des témoignages documentaires, des visuels " photographiques ». Quelque chose qui relève, en définitive, d'une archive tout en problématisant le statut de celle-ci puisqu'il s'agit d'œuvres picturales et donc d'art.

Dans Place des héros, le regard que porte la veuve Schuster à travers la vitre de la fenêtre de l'appartement en surplomb de la place historique n'est pas dirigé. Elle ne voit rien, mais

tons d'ailleurs que la théorisation des formes contemporaines (lesquelles font entorse pour partie à ces invariants) se fonde sur la liquéfaction et la déconstruction de ceux-ci. 
elle entend encore les clameurs vibrantes de la foule de 1938. L'archive sonore et historique contribue à actualiser cet événement plus de cinquante ans plus tard. Tout séquençage temporel est floué. Aucune image n'est produite, mais l'image acoustique rend co-présent des temps différents de l'Histoire.

Dans Soubresaut, l'espace scénique partiellement en chantier, déconstruit, pulvérisé, pris dans le chaos que nourrissent des comédiens et comédiennes en perpétuel déséquilibre physique et vocal, fait sourdre des bruits de bombardements lointains. Des bruits, suffisamment distincts, mais pas encombrants, se mêlent ainsi à des mouvements symphoniques de percussions... au point que l'union de l'un et de l'autre forme un alliage impromptu, un inouï inattendu propre à rappeler les expériences sonores de la « musique concrète ».

D'évidence, ces trois exemples conduisent à deux types de remarques.

Dans un premier temps, et dans un rapport de simplicité à ce qui est proposé au moment de la représentation, il est toujours possible de voir dans ces images - visuelles et acoustiques - un ensemble de constituants qui structure la fiction comme un espace de redoublement du monde extérieur. Effet miroir, disons-nous, où la gravure et le son se déploient dans l'espace théâtral comme un écho qui a son origine, et s'est donc formé, en un temps et un lieu antérieurs à la représentation. Une origine localisée dans l'Histoire.

Comme tout « écho », pour autant qu'il fait entendre son foyer d'origine, il parvient également sous une forme plus ou moins différente de la référence originale. C'estle propre du travail poétique, plastique, esthétique de faire de l'écho, tout à la fois ce qui ressemble à la référence originale et ce qui s'en distingue. Et c'est bien entendu dans l'écart qu'est l'écho que l'original s'observe différemment. Tout travail théâtral, qui repose sur cette pratique de la ressemblance et de la dissemblance, constitue donc l'espace théâtral comme un miroir où le principe de reconnaissance permet de gagner une connaissance accrue de l'original.

Via le recours à des archives et des documents historiques : les gravures de la guerre de Trente ans (dans un rapport parabolique typiquement brechtien) ; l'enregistrement de 1938 des clameurs de la Place des héros à Vienne, la bande son des bombardements dans Soubresaut... l'espace théâtral est ainsi fondé sur un rapport dialectique entre dedans et dehors, un espacement entre modèle référentiel et modèle dérivé. Images sonores et visuelles favorisent dès lors un lien optique à ce qui est présenté.

Dit autrement, ce qui est vu/entendu sur la scène est identifié via la mémoire que l'on a de l'extérieur. Le travail théâtral conduit dès lors à une actualisation et une esthétique du témoignage ; et la scène, elle, à un divan où les motifs pulsionnels (affectifs, mémoriels, sociétaux) émis du plateau convoquent le souvenir.

Mais d'un autre point de vue, si l'espace théâtral est pour partie le lieu de la convocation, la complexité plastique du phénomène théâtral n'est pas sans produire des foyers de résistance. Le temps de la représentation et sa structure ne relèvent donc pas exclusivement d'un théâtre épiphylogénétique : un « dépôt de mémoire ${ }^{13}$, mais aussi de l'expérience esthétique que fait le sujet. C'est-à-dire d'une épreuve, où simultanément à la faculté optique (voir/reconnaitre/se souvenir), la fonction haptique (qui est une puissance seconde de l'optique) est mise en mouvement. Par

13 lbid., p. 77 
fonction haptique, entendons une " possibilité du regard » qui loin d'être subordonnée à la vision et arrêtée par la matière visuelle, passe par celle-ci, la traverse et accède à un espace tactile. C'est-à-dire un monde d'impressions et de dépôt de sensations qui renvoie l'espace matériel à une peau qui enveloppe celui-ci et abrite un espace non matérialisé, non référencé. On passe ainsi du « voir » (esthétique de la réception) au « donner à voir » (phénoménologie de la perception).

La puissance du phénomène théâtral est donc d'être, tout à la fois, espace de convocation et espace de conduction ou de connexion. Et, en définitive, si le théâtre s'apparente à une chambre d'écho, il est aussi en son fonctionnement un « point d'appui » et une « ligne de fuite » pour celui qui est en situation de spectateur. C'est-à-dire, et pour le formuler à la manière de Gilles Deleuze qui désigne là l'activité de la pensée, « une ligne de sorcière $»^{14}$.

Aussi, regarder les gravures de la guerre de Trente ans de Mère Courage, écouter l'archive sonore des clameurs de 1938 de la Place des héros, distinguer les vagues de bombardement via une bande son documentaire dans Soubresaut... peut nous maintenir à l'endroit d'un commentaire qui s'inquiétera du sens et de la signification de ces épisodes dans le processus de création, et de leurs effets sur notre compréhension de l'Histoire. Ces éléments peuvent, de fait, nous amener à réfléchir sur la nature de ces gestes théâtraux et sur l'identité de ces pratiques théâtrales (réalistes, naturalistes, documentaires...).

Mais, et aussi, d'une manière plus aléatoire mais tout aussi juste, le glissement de l'activité optique à l'activité haptique peut aussi nous

14 Deleuze, Gilles. Pourparlers. Paris: Minuit, 1990. p. 141 conduire à repenser l'enjeu de l'acte théâtral qui, d'un point de vue structurel, entretient toujours des points de tangence, des zones de contiguité, des territoires d'intimité... avec ce qui excède le théâtre et s'appelle la vie.

Dès lors, l'expérience esthétique que fait le sujet, loin de figurer une fin, n'est qu'une étape nécessaire ou un passage qui relève d'une forme d'épaississement du temps et de l'espace propice à l'expérience de la pensée de soi/sur soi. Temps et espace qui, simultanément à la représentation, dans le temps de la représentation, n'appartiennent pas à la représentation, mais se conçoivent comme une faille et une ouverture donnant sur le battement de la vie. Dans cet espace-temps prend forme une herméneutique du sujet qui tient à un processus de subjectivation ${ }^{15}$ qui est le propre d'une expérience ${ }^{16}$ intensive.

Au terme de ces remarques, il me semble alors que le phénomène théâtral - qui met en balance une «culture de soi » et une économie de la culture (cf. la « communauté sensible») - est le lieu d'un enjeu qui tend à émanciper celui-ci de la visée d'un ordre esthético-politique dominant qui n'intègre pas la dimension de l'herméneutique du sujet. Et d'ajouter que les pratiques théâtrales urbaines en espace ouvert et public sont l'horizon de cette herméneutique, l'horizon de ces nouveaux branchements qui rompent, comme le dénoncent Horkheimer et Adorno dès les années 30, avec « la standardisation et la production en série

15 C'est-à-dire la découverte d'une pensée, d'un impensé qui a à voir avec « la constitution de modes d'existence [...] l'invention de nouvelles possibilités ». In: Deleuze, Gilles. Pourparlers... op.cit., p. 131.

16 Ce qui est au juste la pensée. Rappelons encore Deleuze à ce propos : " Penser, c'est toujours expérimenter, non pas interpréter, mais expérimenter, et l'expérimentation, c'est toujours l'actuel, le naissant, le nouveau, ce qui est en train de se faire ». Ibid., p. 144. 
${ }^{17}$, « l'appauvrissement des matériaux esthétiques ${ }^{18}$, l'organisation du marché de l'offre et de la demande qui consiste à encourager le jeu et les attentes du système social au détriment de l'œuvre et du sujet. Soit une rupture, in fine, avec « l'industrie culturelle (qui) ne sublime pas, mais réprime ${ }^{19}$, et produit " sans cesse de nouveaux effets qui restent conformes à l'ancien modèle, (et) sert uniquement à augmenter le pouvoir de conventions $»^{20}$.

\section{Ordre public versus pratique} « sauvage».

Avant de poursuivre, une note liminaire préventive est nécessaire, car il sera toujours possible de penser que notre propos s'apparente à une critique opposant une pratique théâtrale à une autre pratique, qui se déploie en espaces ouverts, laquelle emporterait notre suffrage.

II n'est pas question ici, d'aucune manière, de dresser un tableau ou un palmarès de ce qui est souhaitable ou non, de ce qui serait juste et pertinent, d'un théâtre qu'il faudrait privilégier ou pas.

Notre réflexion et notre exposé ne rendent pas compte de la multiplicité des expériences théâtrales et nous interdisent ce type de rapport et d'évaluation.

L'examen auquel nous avons procédé jusqu'à maintenant a essayé simplement de définir une tendance et une orientation qui, via la reconduction d'invariants structurels, maintiennent chez le spectateur un « horizon d'at-

17 Horkheimer, Max; Adorno, Theodor. La Dialectique de la raison. Trad. Eliane Kaufholz. Paris: Gallimard, 1974. p. 130.

18 Ibid., p. 133.

19 Ibid., p. 148.

20 Ibid., p. 137. tente » comme le commenterait Paul Ricoeur, voire conduiraient à une totémisation et fétichisation du phénomène théâtral. A partir de cela, et pour les raisons que nous avons soulignées, s'esquisse l'idée que le projet esthético-politique de « communauté sensible » s'apparente en définitive à une « conquête de l'audience » qui, comme Jean-François Lyotard le souligne, s'appelait en d'autres temps une « doxa $»^{21}$. À partir de là, également, la pensée d'Horkheimer et Adorno jusqu'à celle de Frédéric Jameson sur « l'industrialisation culturelle » ou Guy Debord sur la « société du spectacle » est difficilement contournable.

Si notre exposé est donc critique, il l'est à l'endroit des finalités et des visées de ces pratiques théâtrales qui, sous la contrainte et la conjonction d'un ensemble idéologico-économique ${ }^{22}$, sont finalement, à leur insu ou pas, au service de l'intérêt et du souci du « faire communauté ». Soit, et pour envisager cela au prisme de l'esthétique, le développement de pratiques théâtrales qui reposent et favorisent le dialogue (donc a priori deux espaces d'énonciation qui reprennent la schize que nous avons posée entre la référence et le modèle dérivé) plutôt que le soli-logue (le dialogue avec soi-même). Et ce parce que, soulignons-le encore, l'organisation structurelle du théâtre (aussi bien les constituants théâtraux que l'industrie du spectacle) participe d'un programme de « fabrique du commun » où est préférée la notion de pu-

21 Jean-François Lyotard, Textes dispersé I : esthétique et théorie de l'art, Leuven University Press, 2012, p. 176. Lire le chapitre «Arraisonnement de l'art. Epockhè de la communication ». p. 176-192.

22 II va sans dire que les esthétiques théâtrales, dans leur diversité et leur pluralité, répondront dès lors à des injonctions idéologiques qui organisent la société du spectacle. Ou, pour le formuler sur le mode lyotardien, il s'agira de produire des œuvres " programmables » obéissant en cela au « programme » esthético-politique. Ibid., p. 176. 
blic (et donc de peuple) à la notion de sujet (et donc de singularité quelconque).

À cette organisation tactique du phénomène théâtral, les pratiques en espaces ouverts seront alors considérées comme une alternative.

Alternative à l'uniformisation et à l'instrumentalisation d'un art, alternative et foyer de résistance aux modes de contrôle, alternative aux espaces d'enfermement que sont trop souvent les théâtres, alternative à un théâtre de communication et de transmission ${ }^{23}$, alternative à l'industrialisation du phénomène et du langage théâtral, alternative à la scénographie du révélé propre à l'espace dialectique scène/ salle et ses variations, alternative au pragmatisme qu'impose les " assis de la pensée ", alternative à une société du spectacle parfaitement organisée, alternative à l'administration du phénomène théâtral, alternative à l'aliénation des pratiques artistiques au modèle d'organisation et de législation du travail, alternative à la production en série et à la mode, au régime marchand et industriel, alternative à l'effet et à la seule visée de construction de « communauté sensible ».

Soit une alternative incarnant un modèle de « biopolitique ${ }^{24}$ qui a pour objet de libérer la vie, de la soustraire au dressage institutionnel tel qu'il a pu être commenté dans La Volonté de savoir par Michel Foucault lorsqu'il analyse le « bio-pouvoir ».

Dès lors, et à ce stade de notre réflexion, il nous faut encore préciser que ce qui est en cause, via ces alternatives, ne s'oppose pas à la portée ou à la visée critique des pratiques

23 Gilles Deleuze définit les sociétés de contrôle en soulignant qu'elles reposent sur la circulation de l'information et de la communication. Une société de contrôle se fonde donc sur la propagation de mots d'ordre.

24 Negri, Antonio. Du Retour. Abécédaire biopolitique. Entretie avec Anne Dufourmontelle. Paris: Calmann-Lévy, 2002. p. 89. théâtrales en espaces fermés. Nombre de spectacles, de dispositifs, de mises en scène... sont explicitement inscrits dans un rapport critique et poétique qui prend forme sous diverses esthétiques. Ce n'est pas davantage l'enjeu du plaisir, de l'intensité, de l'émotion, de la sensation vive... nés de ces formes qui est en cause. Encore une fois, et martelons-le, aucun anathème n'est jeté sur ces créations. Sur le mode de Perec, nombre d'entre elles mériteraient que le témoin de celles-ci ouvre un carnet du spectateur critique qui commencerait par « Je me souviens »...

Mais, à l'avantage des pratiques théâtrales en espaces ouverts, si on peut les créditer d'une résistance aux modes de contrôle que nous avons soulignés, elles sont aussi, et surtout, le ferment et l'agent d'une autre pensée qui, tout en procédant d'esthétiques diverses ${ }^{25}$, recoupent des enjeux anthropologiques et politiques. Car d'évidence, les praticiens du théâtre en espaces ouverts sont dans un rapport critique et théorique à l'espace. Ils sont dans un rapport d'analyse à l'organisation spatiale de l'État qui se déploie sous le modèle d'une société : ses mouvements, ses flux, ses zones de contrôle, ses périmètres localisés et divisés, ses territoires réglés par le droit et la loi qui constituent l'espace public comme, et d'abord, un ordre public.

Or cet ordre, comme de nombreux penseurs en font la critique, relève d'une institution imaginaire de la société (cf. Cornelius Castoriadis). Il participe en cela, exclusivement, d'un choix arbitraire qui se fonde sur l'exclusion autoritaire d'autres possibilités. II procède surtout, in fine, d'un agencement qui repose sur un rapport idéologico-économique où, l'exposition

25 On parlera facilement de «formes performatives » pour désigner ces pratiques en espaces ouverts. 
de l'art entre autres, se contemple comme un ordre esthétique réfléchissant l'ordre politique dominant.

Dans un rapport critique aigu à l'organisation de cette société divisée - et « toute société divisée est une société de servitude ${ }^{26}$ écrit Pierre Clastres ; dans un rapport de défiance à l'organisation du spectacle - et « le spectacle est le discours ininterrompu que l'ordre présent tient sur lui-même, son monologue élogieux. C'est l'auto-portrait du pouvoir à l'époque de sa gestion totalitaire des conditions d'existence ${ }^{27}$ écrit Guy Debord ; dans un rapport de soupçon vis-à-vis des « équipements » qui saturent l'espace - comme le commente Felix Guattari à propos des territoires urbains : « La ville est la structure totalisant les équipements, eux-mêmes machines du socius. La ville est le seuil de densités des machines du socius ${ }^{28}$; dans un rapport conflictuel à la main mise de l'État et du pouvoir sur « l'espace public [...] complètement évacué en régime capitaliste ${ }^{29}$ comme le répond Gilles Deleuze à Michel Foucault qui, lui, rappelle qu'un « rapport de pouvoir sous-tend l'existence de l'équipement collectif et son fonctionnement $" 30$ tout en dénonçant la visée prescriptive de l'espace public qui n'est plus un espace de production mais un territoire de consommation normalisée ${ }^{31} \ldots$ les

26 Clastres, Pierre. Une Société contre l'État. Recherche d'anthropologie politique. Paris: Minuit, 1974. p. 113.

27 Debord, Guy. La Société... op.cit., p. 12.

28 « Premières discussions, premiers balbutiements : la ville est-elle une force productive ou d'antiproduction ? » entretien F. Fourquet et F. Guattari, Les Équipements du pouvoir, décembre 1973, pp. 27-31. In: Foucault, Michel. Dits et écrits 1, 1954-1975. Paris: Gallimard, 2001. p. 1316.

29 Ibid., p. 1323.

30 Ibid., p. 1319.

31 Or la disparition de cette activité productive (c'est-à-dire les espaces d'invention, d'action et d'imagination propres à l'individu) au bénéfice de la consommation normalisée n'ap-
« acteurs » des pratiques en espaces ouverts ont un lien différent à la contamination de l'espace public par la seule logique libérale développant un mode de production sélectif et exclusif qui ne tend qu'à favoriser une demande uniformisée ${ }^{32}$.

Demande qui, par ailleurs, satisfait une classe dominante en excluant le plus grand nombre, entretenant une séparation, une division, voire une getthoïsation au sein même du champ social. Soit un paradoxe qui semble ne plus interpeller le pouvoir dont l'action, bien qu'elle se réclame de la construction d'une « communauté sensible » ou du souci du «Mit sein » (l'être-ensemble), s'arrange d'un effet social où le «Mit welt » (le monde en commun) ne relève plus que d'une chimère.

Aussi, au modèle de société qui s'est développé sous l'influence libérale (division, saturation, découpage, nomenclature, fléchage, mise aux normes, privatisation... de l'espace...), devant cette «mondialité » conceptualisée par Henri Lefebvre dans La Production de l'espace, face à la « compacité » que relève Miguel Abensour (c'est-à-dire la disparition physique d'espaces disponibles ou la surabondance d'espaces réglés), l'intervention, l'introduction ou l'émergence d'énergies différentielles comme peuvent le représenter les actions des artistes en espaces ouverts sont nécessairement perçues comme des mouvements de fragilisation, de contestation et de déstabilisation

pelle d'autre commentaire que celui de Guattari : « l'essence de la ville despotique est son activité d'antiproduction ", ibid., p. 1317. Dans le même ordre d'idée qui souligne la contiguité entre l'économie libérale et une société de contrôle, Debord le rejoint : " le spectacle est le moment où la marchandise est parvenue à l'occupation totale de la vie sociale [...] La production économique étend sa dictature extensivement et intensivement », in G. Debord, La Société... op.cit., p. 25.

32 Rien n'est plus édifiant que de se pencher sur l'organisation de la circulation des « œuvres » pour s'assurer de cette structuration et de cette organisation. 
d'un espace occupé et pensé a priori comme légitime. "A priori », car, et n'en doutons pas, la visée du modèle libéral est bien, comme le rappelle Jean-François Lyotard, de " détruire ce qui reste des cultures non capitalistes ${ }^{33}$. Et pour autant que l'apparente et fragile harmonie est entretenue et règle les relations du corps social, comme l'écrit Foucault il n'y a là qu'une illusion fabriquée par le déploiement des modesde contrôle :« Je crois que le grand fantasme, c'est l'idée d'un corps social qui serait constitué par l'universalité des volontés. Or ce n'est pas le consensus qui fait apparaître le corps social, c'est la matérialité du pouvoir sur le corps même des individus $»^{34}$.

Dès lors, alors que les manifestations des pratiques artistiques en espaces ouverts ne sont rien moins qu'un élan vital (au sens bergsonien) et légitime puisque l'espace urbain, comme le pense Lefebvre, est le lieu et l'enjeu de rapports sociaux qui peuvent induire des tensions, des tentatives de réappropriation et des conflits (y compris esthétiques), elles seront perçues par le Politique comme des perturbations et des « violences » faites à l'ordre public. À l'endroit du spectacle, les productions dans l'espace ouvert se percevront donc comme une entorse et un défi à l'ordre esthétique puisque « le spectacle [...] est la représentation diplomatique de la société hiérarchique devant elle-même, ou toute autre parole est bannie »35 commentait Debord. Mais, et nous devons en garantir la visibilité et la conscience, rien de l'ordre établi et privilégié par le Politique, rien des institutions dominantes, rien des différents modes de contrôle mis en place ne suffit à légi-

33 Lyotard, Jean-François. Des Dispositifs pulsionnels. Paris: Galilée, 1994. p. 13.

34 Foucault, Michel. Dits et Ecrits 1... op. cit., p. 1622.

35 Debord, Guy. La Société du spectacle... op. cit., p. 11. timer l'ordre, ni même à masquer la seule évidence logique que rappelle Georges Balandier : « l'ordre et le désordre sont comme l'avers et le revers d'une monnaie : indissociables ${ }^{36}$. Soit, et pour le formuler à la manière de René Char, un flou entre « la base et le sommet ${ }^{37}$ qui sont dans un rapport de contiguïté, de mouvements et parfois d'inversion des représentations comme peut l'être L'América invertida (1943) de Joaquin Torres Garcia.

Aussi, et en définitive, les pratiques artistiques en espaces ouverts ne sont pas plus ou moins légitimes que celles qui sont l'apanage des stratégies et des tactiques de l'ordre établi qui participe de ce que l'on pourrait appeler le «banditisme des sociétés ${ }^{38}$. Si elles paraissent incarner des formes menaçantes, des mouvements d'insurrections sociales, si elles contreviennent au consensus, si en cela elles rappellent que l'espace public est dissensuel et qu'elles portent atteinte, en les dissolvant, aux repères de la certitude ; elles s'apparentent à de petits séismes, " à des sources de chaleur sous la surface froide de la société ${ }^{39}$, des foyers d'intensité de vie qui s'élabore à la marge de l'ordre... En cela, elles sont « une sorte de secousse géologique ${ }^{40}$.

A l'idéologie dominante qui a pris la forme d'un corps social figé et domestiqué (y compris donc les formes esthétiques promues), elles répondent ainsi en réintroduisant un geste exclu, un corps physique et artistique. Et il n'y a rien à cet endroit qui ressemble à

36 Balandier, Georges. Le Désordre. éloge du mouvement. Paris: Fayard, 1988. p. 117.

37 Char, René. Recherche de la base et du sommet. Paris:Gallimard, 1971.

38 Ibid., p. 31.

39 Balandier, Georges. Le Désordre...op.cit., p. 74.

40 Debord, Guy. La Société du spectacle... op. cit., p. 11. 
une reconquête illégale de l'espace, rien qui soit contre-nature, puisque comme l'analysent Gilles Deleuze et Felix Guattari à propos des espaces lisses et striés, ces « deux espaces n'existent en fait que par leurs mélanges l'un avec l'autre : l'espace lisse ne cesse pas d'être traduit, transversé dans un espace strié ; l'espace strié est constamment reversé, rendu à l'espace lisse ${ }^{41}$.

Le dérèglement ou le désordre auquel sera assimilée intempestivement une pratique artistique alternative remettant en cause l'ordre esthétique n'est donc en définitive qu'une force légitime. Non pas un désordre, mais un mouvement inhérent à la configuration de l'espace de la Polis qui est indépassablementliée au Polos ${ }^{42}$. Au vrai, il n'y a là que l'exercice d'une « démocratie sauvage » comme l'écrirait Abensour. C'est-à-dire la réintroduction d'une diversité qui favorise l'émergence d'un agorique nécessaire.

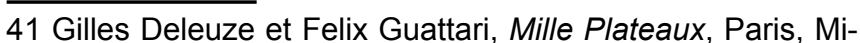
nuit, 1980, p. 593. Un des chapitres du livre est consacré, entre autres, à la compréhension et la représentation de l'espace. Deleuze et Guattari définissent ainsi l'espace strié comme un espace aménagé, réglé, occupé ; alors que l'espace lisse relève lui de frontières et de limites incertaines. Ils développent l'idée que l'un et l'autre des espaces sont constamment dans un lien de conflictualité, un mouvement de flux et de reflux. Par une sorte de jeu agonal, d'énergies et de forces qui s'opposent, espaces lisses et striés sont ainsi dans une relation dialectique où ce qui a été sédentarisé, domestiqué, figé... ne peut s'apparenter qu'à un état provisoire, jamais définitif, puisque l'espace est soumis simultanément à la nomadisation, au sauvage, au mouvement. D'une certaine manière, et par exemple, le modèle démocratique installé et institué via l'appareil d'État n'est pas à l'abri de ce Miguel Abensour appelle les formes de "démocraties sauvages ». C'est-à-dire la réappropriation, par la lutte, d'un espace conquis et fermé qui, à nouveau, peut re-devenir un horizon ouvert.

42 Polos et Polis viennent de politeia qui signifie « art de gouverner la cité ». L'idée de "Polis » grecque, celle qui a nourri nos représentations d'un modèle de développement politique et d'organisation de l'espace public, n'est pas séparable, étymologiquement, du "Polos » qui vient de "Peleîn » : " tourner autour d'un axe ». Ainsi, la Polis, dérivée du Polos, recouvrerait enfin son mouvement qui, comme l'interprète Philippe Ivernel dans Le Tournant esthétique, est d'abord « tourbillon », et donc mouvement.
En guise de suspension, il faut alors admettre que la coprésence de formes artistiques, relevant pour les unes de l'ordre établi, pour les autres de pratiques alternatives en espaces ouverts, entre en concurrence. Cela étant, valider la nature concurrentielle de ces spectacles revient à maintenir le paradigme de l'offre et de la demande, et par là, conforter la pensée de Guy Debord lorsqu'il analyse les travers du spectaculaire « la racine du spectacle est dans le terrain de l'économie devenue abondante, et c'est de là que viennent les fruits qui tendent finalement à dominer le marché spectaculaire ${ }^{43}$. Mais ce seul commentaire qui vaut davantage pour un constat que pour une analyse ne suffit à éclairer ce qui est en jeu.

Or ce qui est en jeu ne relève d'aucune autre topique que celle qui concerne l'écriture de l'espace qui renvoie à celle de l'Histoire. Soit, et précisons-le, un geste d'écriture qui se confond avec un geste politique.

Dès lors, si l'ordre établi et les équipements qui le relaient relèvent d'une pratique d'écriture - que d'aucuns identifient à " un flux d'écriture despotique ${ }^{44}$ - les pratiques artistiques en espaces ouverts sont la tentative politique, et notamment esthétique, de contrer cette écriture - « ces faux en écriture » - en écrivant autrement les rapports qui se tissent dans l'espace. D'une certaine manière, et avant de poursuivre, nous dirons en conséquence que l'appréhension des problématiques spatiales et la compréhension des enjeux liés à la structuration de l'espace induisent une réflexion sur l'écriture, le langage et ses épiphénomènes ; au

43 Debord, Guy. La Société du spectacle... op. cit., p. 11. Déclinant la nature de ce phénomène, Guy Debord parlera également de « liberté dictoriale du Marché ». id.

44 C'est ainsi que Felix Guattari en parle. In: Foucault, Michel. Dits et écrits $1 \ldots$ op. cit., p. 1316. 
point que les pratiques artistiques en espaces ouverts peuvent être identifiées à une réponse au questionnement d'Eduardo Galeano sur le modèle de société privilégié, entre autres, en Amérique du Sud. Je cite : «Que leur offre la voix du système ? Le système parle un langage surréaliste $»^{45}$.

\section{« Le bas langage ${ }^{46}$}

On pourrait s'étonner du titre par lequel nous ouvrons notre propos à l'endroit du langage. Dissipons tout d'abord les zones d'ambiguïté que pourrait induire cette « expression ».

Recourant à celle-ci, il n'y a aucune connotation péjorative. Si nous parlons de « bas langage ", c'est que les pratiques artistiques en espaces ouverts recourent bien souvent à des formes de langage qui les distinguent du phénomène théâtral tel qu'il est produit en espace clôt. Or - et il n'est pas besoin de développer sur ce point - le phénomène théâtral en espace clôt se fonde sur « le langage du spectacle (qui) est constitué par des signes de la production régnante, qui sont en même temps la finalité dernière de cette production ${ }^{47}$.

45 Galeano, Eduardo. Les Veines ouvertes d'Amérique latine. Trad.: C. Couffon. Paris: Plon, 1981. p. 16.

46 Walter Benjamin, « Problèmes de sociologie du langage », OEuvres III, trad. M.Candillac, R. Rochlitz et P. Rusch, Paris, Gallimard, 2000, p. 21. Benjamin cite là une expression d'Alfredo Niceforo, auteur de Le Génie de l'argot. Essai sur les langages spéciaux, les argots et les parlers magiques. " Le bas langage " benjaminien, précisons-le, n'a rien à voir avec le " discours d'en haut » auquel il s'opposerait comme l'évoque Jacques Rancière dans La Parole ouvrière.

47 Debord, Guy. La Société ... op. cit., p. 5. Au nombre des constituants de ce langage, on citera : le théâtre de texte (répertoire et partrimonial), l'architecture des édifices théâtraux, les constituants scéniques du spectacle, les conventions théâtrales autant que sociales, etc. qui obéissent à une logique d'identification du phénomène théâtral dans un espace social cloisonné et procèdent d'une organisation tactique, socio-economique, réfléchissant un ordre politique et idéologique.
Par « bas langage », et avant d'en préciser le contenu, nous faisons référence en définitive à une organisation verticale de l'espace sociétal qui privilégie des formes de visibilité dans lesquels elle s'incarne et qu'elle revendique comme les traits propres d'une société. Au visible, à la centralité, au soutenu, à ce qui fait symptôme... correspond donc, toujours dans cette configuration verticale, d'autres formes, invisibles, marginales, fragiles, paupérisées. Soit un écart entre « la base et le sommet »...

Par « bas langage » nous désignons donc ce qui, tout en étant dans l'espace sociétal, constitue « la forêt des délaissés urbains et humains ${ }^{48}$, laquelle peut figurer « un enjeu social, économique mais aussi esthétique » pour reprendre une formule de l'architecte en urbanisme Patrick Bouchain ${ }^{49}$.

C'est-à-dire, et nous nous inscrivons dans le prolongement de la pensée de Bouchain, que les espaces délaissés et résiduels peuvent être considérés comme des laboratoires à partir desquelles s'imaginent des politiques alternatives puisqu'ils sont hors jeu du marché, du moins jusqu'à ce l'ordre établi « exproprie » de ces espaces ou interdisent ceux-ci, à ceux qui les investissent, par exemple les communau-

48 Guillaume, Maurice, L'archipel des délaissés. Critique, n. 757-758, p. 542-555, Paris, Minuit, 2010. Article consacré à la pensée de Patrick Bouchain. Dans le langage qu'utilisent les techniciens et les penseurs de l'espace, un « délaissé » est un espace qui n'a aucune fonction, souvent abandonné. Ce que Bouchain appelle un « impensé de la ville ».

49 Patrick Bouchain évoque sa pratique d'architecte comme un « travail de metteur en scène », considérant « que l'architecture est politique et qu'elle doit répondre au souci de l'intérêt général » comme il le confiait à Michel Leloup (cf. « Patrick Bouchain, pour faire avancer l'architecture, il faut de l'audace », l'Express, 13 juin 2005). II est un pionnier du réaménagement de lieux industriels en espaces culturels (La Friche Belle de Mai à Marseille)... entre autres. Comme le souligne cet ancien conseiller (1988-1995) auprès du Ministre de la culture Jacques Lang, P. Bouchain articule projet urbain et population, en observant un enseignement H.Q.H. (Haute qualité humaine). 
tés de pratiques artistiques alternatives.

Or les espaces délaissés et résiduels sont légions dans une société qui, régie par la logique du marché du spectacle, a concentré, isolé et organisé les espaces où se donne le phénomène théâtral. Ils s'apparentent à tous les territoires qui n'ont pas été homologués par les gardiens du spectaculaire et la police esthétique : rues, places, ponts, terrains vagues, halles, squares, jardins publics, arrêts de bus, etc.

Aux lieux dévolus au phénomène théâtral, aux distances qui organisent les déplacements dans l'espace urbain, aux « cadastres » fonctionnalistes, à la signalétique qui flèche les destinations et organise l'information, distribue les concentrations de population et canalise le mouvement... les pratiques artistiques alternatives augmentent l'espace ${ }^{50}$ et le décloisonnent. $\mathrm{Ou}$, et il est plus judicieux de le formuler autrement, elles créent des situations, inventent des espacements, réduisent la distance entre la pratique artistique et les individus qui peuplent l'espace, au point de développer l'idée que si l'homme habite l'espace, l'homme est habité également par l'espace.

C'est qu'à la manière de Martin Heidegger, dans sa conférence Bâtir, Habiter, Penser donnée en 1955 dans le cadre du second entretien de Darmstadt sur "l'homme et l'espace ", la séparation de l'espace et de l'homme n'est pas recevable. Comme l'écrit le philosophe à propos de la relation qui unit l'homme à l'espace, « l'espace n'est pas pour l'homme un vis-à-vis. II n'est ni un objet extérieur ni une expérience limite. II n'y a pas les hommes et en

50 II nous faudrait bien entendu pouvoir développer également, simultanément à notre propos sur l'espace, une réflexion sur le temps et la temporalité des pratiques artistiques alternatives. La façon qu'elles ont de rompre avec la convention de la « soirée », entre autres. plus l'espace ${ }^{51}$.

Cette nuance, qui vaut pour remise en cause de l'esprit cadastral qui gouverne à l'organisation et à la rationalisation de l'espace, confère aux pratiques artistiques alternatives un supplément d'intérêt.

Si elles peuvent être perçues comme des pratiques « rebelles ", voire des manifestations conflictuelles, elles procèdent d'un esprit différent au sens où, apparaissant dans les « non-lieux » de l'espace de l'ordre établi, elles reviennent sur la logique de division privilégiée. Elles renouvellent le rapport fonctionnel à l'espace et l'attribution-articulation d'un lieu limité à une fonction. Par là, elles régénèrent l'enjeu et les formes esthétiques du phénomène théâtral.

En définitive, les pratiques artistiques alternatives corrigent les déficits d'un espace sociétal où la notion de cadastre s'est substituée à celle d'espace, où la fonctionnalité prime sur l'idée d'habitat au sens heidegerrien : «"Les mortels sont", cela veut dire: habitant, ils se tiennent d'un bout à l'autre des espaces, du fait qu'ils séjournent parmi les choses et les lieux ${ }^{52}$. Les pratiques artistiques alternatives qui se déploient, en liberté, ont donc un lien direct avec l'idée de « monde en commun ». Elles le rappellent comme un fondement indépassable de «l'être-ensemble ».

Dès lors, elles figurent effectivement des pratiques de résistance à ce que René Char décrivait : « Le monde contemporain nous a déjà retiré le dialogue, la liberté, l'espérance, les jeux et le bonheur. II s'apprête à descendre au cœur même de notre vie pour éteindre le

51 Heidegger, Martin. Bâtir Habiter Penser. Essais et conférences. Trad.: A. Préau et J. Beauffret. Paris: Gallimard, 1980. p. 186.

52 Ibid., p. 187. 
dernier foyer, celui de la rencontre ${ }^{53}$.

À titre d'exemple, regardant le travail du grupo de théâtre Falos et Stercus dirigé par Marcelo Restori, à Porto Alegre, notamment IIha dos amores, um dialogo sensual com a cidade, en 2013 ; plusieurs indices nous sont donnés sur l'enjeu et la nécessité de la rencontre.

Un groupe de performeurs (comme ils se désignent) envahit la « voie publique », les berges d'un canal pollué qui borde la rivière Arroio Diluvio, le pont ${ }^{54}$ où passent les automobilistes ralentis par la présence de l'action artistique. Aux cris, aux chants, aux mouvements chorégraphiés, à la nudité partielle exposée - qui tiennent les passants à distance- se substituent une rencontre d'un autre ordre.

Quelque chose est ralenti comme mis en suspension. Quelque chose altère le quotidien sans pour autant que celui-ci disparaisse. Quelque chose s'ajoute au temps, au relief, au geste... et l'espace public : l'ordre public, est dessaisi de son ascendance unique sur le mouvement, sur l'organisation de l'espace, sur la répartition des individus au sein de celui-ci, sur les langues qu'on y parle, sur l'écoute et le regard que l'on porte à l'autre...

Et alors que s'exposent un rythme différent, un langage différent, un geste différent, une proximité différente... Alors que cet espace résiduel est investi par les performers, quelque chose d'imprévisible se met en place dans

53 Char, René. Recherche de la base et du sommet... op. cit., p. 35. Selon nous c'est à cet endroit que se loge le clivage entre les espaces de l'ordre établi et ceux occupés par les pratiques artistiques alternatives puisqu'il n'y a, chez les derniers, aucun a priori ni préjugés sur la forme esthétique qui est en jeu dans la rencontre, pas plus qu'il n'y a de présupposés sur la configuration de la rencontre.

54 Pour autant que nous n'avons connaissance des motivations de la localisation de cet acte artistique, on se souviendra des pages d'Heidegger sur cet ouvrage d'art : « le pont rassemble » écrit-il. Heidegger, Martin. Bâtir Habiter Penser... op. cit., p. 181 .. l'espace délaissé.

Un seul épisode, parmi tous les instants qui mêleront passants et performers, sera l'objet de notre propos.

Un père, qui tient son enfant dans ses bras, se rapproche d'une interprète enceinte. L'enfant pose sa main sur le ventre de la jeune femme, puis un échange de paroles à lieu qui n'est pas audible, pas destiné à la communication, mais juste furtif.

Naturellement, cette séquence prélevée de la performance qui recèle bien d'autres éléments pourrait inviter le lecteur à un commentaire sarcastique où l'on arguerait du caractère tactique de cet épisode « humain trop humain ». Mais en l'occurrence ce n'est pas pour sa « valeur humaine » que nous le convoquons, mais plutôt la façon dont structurellement cet « inattendu » recouvre un enjeu tout autant anthropologique qu'esthétique.

Nous appuyant sur cet exemple, il semble que nous puissions convenir que le phénomène théâtral (présence d'un acte artistique dans l'espace ouvert) développant sa propre dynamique esthétique, conjugué à la présence des constituants de l'espace ouvert (architecture, passants, mouvements, forces de police, règlements de l'espace public), produit un phénomène d'hybridation entre deux mondes qui se contaminent et qui se retrouvent pour en former un troisième qui, c'est selon, légitime le propos d'Heidegger et/ou nous inscrit dans un élan qualifié bien souvent d'utopique, alors que la fabrique du commun, le monde en commun et l'être ensemble sont ainsi réels et constatables à travers un geste partagé (cf. l'exemple que nous venons de décrire).

Et d'ajouter que si le théâtre que privilégie le système économico-politique de l'ordre établi prétend avoir pour visée « la communauté 
sensible 》 (expression réductrice qui désigne la production d'une émotion convertissant le public), les pratiques artistiques alternatives fondées sur un éclectisme du brisé semblent, elles, trouver la voie qui permet à « une communauté sans communauté » d'apparaître.

Et ce parce que, peut-être, alors que le théâtre de l'ordre établi repose bien souvent sur un effet miroir où « tout ce qui était directement vécu s'est éloigné dans une représentation „55 conduit à faire de l'instant de la représentation un temps et un objet extérieurs au spectateur (qui renvoie ce dernier à un consumérisme) ; dans le phénomène théâtral nomade la détermination de la pratique artistique n'est pas un reflet second, mais elle est en prise directe avec la société, sa vitalité, son mouvement.

Dès lors, il nous faut tirer un enseignement de tout cela.

Si les différentes pratiques artistiques, les différents phénomènes théâtraux sont, d'un point de vue purement esthétique, complémentaires, qu'ils soient (pour le dire simplement) « dans les murs » ou « hors les murs »; ce qui les oppose c'est la représentation qu'ils donnent du commun, l'image qu'ils en produisent et l'utilisation que le politique peut en faire. Comprenons bien, rien n'oppose directement les expériences esthétiques que nous évoquons.

En revanche, et comme le rappelle Giorgio Agamben qui commente La Société du spectacle de Guy Debord dont il souligne " l'implacable lucidité du diagnostic », «l'économie marchande [...] après avoir falsifié l'ensemble de la production, peut manipuler à présent la perception collective et s'emparer de la mé-

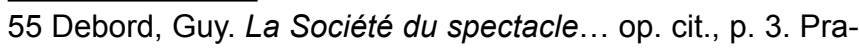
tique qui permet à Guy Debord d'en faire la critique radicale en déclarant que « le spectacle qui inverse le réel est effectivement un produit ». Ibid., p. 6. moire et de la communication sociale, pour les transformer en une unique marchandise spectaculaire, où tout peut être remis en question, sauf le spectacle même qui, en soi, ne dit rien d'autre que : " Ce qui apparaît est bon, et ce qui est bon apparaît " „56.

L'apparition d'une autre image, via les pratiques artistiques alternatives, est donc une infraction dont l'effet est d'écarter l'art du désir de consommation qui s'est répandu à mesure que le marché du spectaculaire s'est développé fabriquant une "pensée grasse ${ }^{57}$. C'est-àdire, en fait, une «non pensée ». Or ce qui est en jeu, c'est la « mémoire », « la communication sociale » », etc.

Ce qui est atteint, c'est donc un système et son image, précisément l'image du système qui produisait, indépendamment des individus qui forment la « communauté », une communauté étrangère à elle-même. Ce qui est atteint, c'est en définitive la communication d'un modèle représentatif qui s'avère n'être qu'un modèle idéologique exclusif. Le déploiement des pratiques artistiques alternatives vaut donc pour une «époché de la communication » : une suspension de la communication entendue au sens de l'ordre établi.

Et c'est à cet endroit que le rapport au langage peut être introduit. Le langage comme espace, précisément, puisque « dans le spectacle c'est notre propre nature linguistique qui s'avance vers nous renversée "58. Comprenons par là que le spectacle (Agamben qui reprend

56 Agamben, Giorgio. La Communauté qui vient, théorie de la singularité quelconque. Trad.: Marilène Raiola. Paris: Seuil, 1990. p. 81

57 Baudrillard, Jean. D'un fragment l'autre. Paris: Albin Michel, 2011. p. 37. Baudrillard désigne par cette expression, les esprits repus et fatigués.

58 Agamben, Giorgio. La Communauté qui vient... op.cit., p. 82. 
le mot de « spectacle » à Debord entend désigner par celui-ci le « produit ») nous prive et nous mutilenon seulement de l'espace, mais également de l'expérience du langage pour nous-mêmes.

Qu'entendons-nous par « le langage comme espace »? À quoi prétendons-nous quand nous disons cela?

Convenons tout d'abord que par « langage " nous ne désignons pas seulement les formes discursives.

Si nous pouvons avancer que le langage est espace, c'est parce que l'espace est à part entière un élément constituant du politique et de la politique.Ajoutons alors à ce que nous avons déjà exposé et rappelons nous ce que disait Mikhail Bakhtine :

Chaque mot, nous le savons, se présente comme une arène en réduction où s'entrecroisent et luttent les accents sociaux à orientation contradictoire. Le mot s'avère, dans la bouche de l'individu, le produit de l'interaction vivante des forces sociales ${ }^{59}$. Rappelons-nous aussi la réflexion d'Herbert Marcuse « la syntaxe, la grammaire, le vocabulaire sont des actes moraux et politiques ${ }^{60}$ ou encore l'analyse d'Hannah Arendt : " dès que le rôle du langage est en jeu, le problème devient politique par définition $» 61$. (Bakhtine, 1977, p. 67).

Le langage est donc à part entière un territoire de conflits, de conquêtes et un enjeu qui, pour l'ordre, consiste à assoir un contrôle via le langage, quand pour les pratiques artistiques alternatives (cf. Le bas langage) il s'agit d'en

59 Bakhtine, Mikhail. Le marxisme et la philosophie du langage, trad. Marina Ya guello. Paris: Minuit, 1977, p. 67.

60 Marcuse, Herbert. L'Homme unidimensionnel, essai sur l'idéologie de la société industrielle avancée. Paris: Minuit, 1968. p. 220

61 Hannah, Arendt. L'Humaine condition. Paris: Gallimard, 2012, p. 61. faire l'expérience en travaillant à une réécriture de l'espace. Ce constat, rapporté à l'art et au phénomène théâtral, nous conduit à convoquer l'analyse d'Herbert Marcuse qui fait écho à celle de Roland Barthes quant à l'utilisation et l'organisation du langage dans l'espace sociétal et son rapport aux arts.

Dans un article intitulé « La Face baroque »62, Roland Barthes montrait que « l'écriture peut tout faire d'une langue, et en premier lieu lui rendre sa liberté ${ }^{63}$. L'étude qu'il fait le conduit alors à interroger les formes littéraires (et théâtrales) soumises aux contraintes. C'est quand il convoque celles-ci qu'il parle de « verbalisme "64 lequel se nomme « plus noblement la poésie ${ }^{65}$. Roland Barthes poursuit en soulignant que la belle œuvre « transforme l'écriture en leçon ${ }^{66}$ la ramenant à une « marchandise utile à l'économie de l'humain ${ }^{67}$, quand le verbalisme ou la poésie génère « l'énergie de parole ${ }^{68}$. Dans le prolongement de cette analyse, Herbert Marcuse, qui cite à plusieurs reprises l'auteur du Bruissement de la langue, partage cette conception, tout en montrant comment le langage, organisé dans le champ social et par ses agents, est dynamité et appauvri par un ensemble de procédures qui l'éloigne de toutes « tensions ». Le matériel linguistique est

62 Barthes, Roland. Le Bruissement de la langue. Essais critiques IV, [s.n.], 1984, Seuil, p. 283-285.

63 Ibid., p. 284.

64 Ibid., p. 285

$65 \mathrm{id}$

66 id.

67 ld. Cette idée liée au registre du commerce est récurrente à l'ensemble de l'article et on la trouve déclinée du début à la fin de «La Face baroque ». Ainsi, au début peut-on lire : « Importe au Français le "quelque chose à dire" ce qu'on désigne couramment d'un mot phonétiquement ambigu, monétaire, commercial et littéraire : le fond (ou le fonds ou les fonds) ". lbid., p. 283.

$68 \mathrm{ld}$. 
alors cerné, « ciselé », mis en conformité avec le rôle qui lui est attribué, et par là privé de tout espace qui pourrait générer la « critique ». Le langage est rendu opérationnel, son usage est fonctionnel, fondé sur le rejet des "éléments non conformes ${ }^{69}$. II définit ainsi ce langage comme un "langage harmonisé qui est fondamentalement anti-critique et anti-dialectique ${ }^{70}$. Le point de vue de Marcuse s'augmente alors d'une réflexion sur les effets du langage fonctionnel. Le développement de celui-ci a ainsi pour principal résultat d'être « radicalement anti-historique » afin de s'écarter des « contenus subversifs de la mémoire ${ }^{71}$.

En d'autres termes, et $\mathrm{H}$. Marcuse le soulignera ultérieurement, les contraintes opérationnelles qui s'exercent sur le langage, mutilent la «forme de médiation $»^{72}$ qu'induit celui-ci et qui est l'une de ses caractéristiques essentielles. Le langage, ramené au « langage clos ${ }^{73}$, repose ainsi sur un principe récurrent à son déploiement qui consiste à brouiller la mémoire. Le sujet devient alors le familier d'une étrangeté au pouvoir et à la force du langage. L'ensemble des composantes du champ social (ce que Louis Althusser ${ }^{74}$ définira ultérieu-

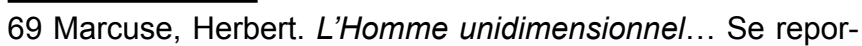
ter à la section de l'essai intitulé "L'univers du discours clos», p. 109-144.

70 Ibid., p. 121.

71 lbid., p. 123.

72 Ibid., p. 123. Marcuse soutient ce raisonnement en convoquant différents penseurs, notamment en citant Adorno : « la société bourgeoise en progrès liquide la mémoire, le temps, le souvenir, en tant que résidus irrationnels $»$, ibid., p. 124. Th. W. Adorno, "Wes bedeutet Aufarbeitung des Vergangenheit ? », in Bericht über die Erziehertkonferenz am 6 und 7 November in Wiesbaden, Francfort, 1960, p. 14.

73 S'il faut définir le « langage clos », rappelons alors la définition qu'en donne Herbert Marcuse : «Le langage clos ne démontre pas, il n'explique pas - il communique la décision, le diktat, l'ordre ». Ibid., p. 126.

74 Louis Althusser décrit, entre autres, que l'histoire de l'espace social est inscrite dans une lutte des classes organisée rement comme les A.R.E. et les A.I.E.) travaille ainsi dans le même but et à la même entreprise où « l'homme est entraîné à oublier » sa faculté critique, le libre exercice de cette faculté par le biais d'un langage hors-tension.

Recoupant les points de vue de R. Barthes et de $\mathrm{H}$. Marcuse, la lecture croisée de l'un et de l'autre nous éclaire sur le rapport que le théâtre privilégié par l'ordre établi entretient au langage clos.

Or, au cours de l'histoire du théâtre, des voix se sont ainsi élevées contre cette subordination qui pétrifiait le langage théâtral dans un ordre poétique et esthétique qui n'était que le prolongement d'un ordre politique. À commencer par celle, retentissante, d'Artaud. Je cite : "La question qui se pose est de permettre au théâtre de retrouver son vrai langage, langage spatial, langage de gestes, d'attitudes, d'expressions et de mimiques, langage des cris et d'onomatopées [...] qui auront autant d'importance intellectuelle et de signification sensible que le langage des mots ${ }^{75}$. Ou encore, « La question du théâtre, c'est poser la question du langage qui n'appartiendrait qu'au théâtre $»^{76}$.

entre un pôle dominant et un pôle dominé. Au service de la classe dominante, il souligne que l'A.R.E (appareil répressif d'État) et les A.I.E. (appareil d'idéologique d'État) permettent d'organiser l'ordre social et de le légitimer, soit en recourant à des agents violents légitimes (police, tribunaux, armées, etc) qui participent de l'A.R.E. ; soit en développant un réseau d'A.I.E. qui sont tout aussi coercitifs mais dont la violence est idéologique. C'est-à-dire qu'elle s'exerce sur les mentalités et la représentation que l'on se fait de soi-même. Au nombre de ces A.I.E, on compte, par exemple, les Lettres et les beauxarts. Soulignons pour finir que les A.R.E relèvent du public, quand les A.I.E. procèdent d'une gestion qui peut être aussi bien publique que privée.

75 Artaud, Antonin. CEuvres complètes, tome V, Paris, Gallimard, 1964. Le volume est sous-titré " Autour du théâtre et son double, et des Censi ». L'édition produit deux fois ce passage : avant la représentation des Censi et après. Le premier sous le titre "Le théâtre que je vais fonder » (p. 35-37). Le second, dans une lettre à Monsieur Van Cauclert, en date du 6 juillet 1932 (p. 101).

76 Ibid., p. 15. 
Ou pour finir, « une conception européenne du théâtre veut que le théâtre soit confondu avec le texte [...]. En face de cela, il nous paraît que [...] la mise en scène devient un langage particulier [...] Ceci conduit à mettre en cause directement le langage de la parole ${ }^{77}$.

Plus tard, on entendra également la voix de Jerzy Grotowski qui ouvre le théâtre à d'autres horizons lors d'une conférence sur « Le Théâtre d'aujourd'hui à la Recherche du rite ${ }^{78}$, le 15 octobre 1968: « J'avais une certaine image du théâtre possible, image qui a été en un certain sens formée en opposition au théâtre existant, ce théâtre trop culturel dans le sens conventionnel, un produit de rencontre de gens cultivés : des gens qui s'occupent avec les mots, les paroles, les gestes, les décors, la lumière, etc., et ensuite d'autres gens bien cultivés qui savent qu'on doit aller au théâtre parce que c'est une sorte d'obligation morale ou culturelle. Au bout du compte tous sortent bien cultivés de ces rencontres, mais rien d'essentiel n'a pu se passer entre ces gens. Chacun reste le prisonnier d'un certain type de conventions, de pensées, d'idées relatives au théâtre ${ }^{79}$.

Perspective, chez Jerzy Grotowski, qui doit permettre d'entrer dans la « voie vers le théâtre vivant » ${ }^{80}$, et ainsi échapper au « théâtre banal $»^{81}$.

D'évidence, alors que les politiques culturelles se mettaient, ici et là, en place, et qu'elles généraient des « mots d'ordre ${ }^{82}$ subsumant

77 lbid., p. 16-17.

78 Le Journal France-Pologne peuples amis, n. 28-29, hiver 1968.

79 lbid., p. 14.

$80 \mathrm{ld}$.

81 lbid., p. 15

82 Celui de "Théâtre populaire », entre autres, qui continue de nourrir les politiques culturelles. la pratique théâtrale à figurer l'un des rouages de tissus urbains de plus en plus complexes, la conscience d'un langage théâtral indépendant des enjeux de la rhétorique politique (soucieuse d'une esthétique relationnelle et de stratégie de communication) s'affirmait donc dans le même temps.

En cela, les pratiques artistiques alternatives que l'on rangera du côté du « verbalisme " s'apparentent de fait à ce qui résiste au « langage clos » puisque, régénérant le phénomène théâtral, elles répondent en définitive à Marcuse comme à Barthes quand il écrit sur le langage théâtral: « qu'est-ce que le théâtraliser ? Ce n'est pas décorer la représentation, c'est illimiter le langage ${ }^{83}$.

En cela, et c'est me semble-t-il le propre des pratiques artistiques alternatives, l'enjeu est de faire exister, en écho à Barthes, un « geste baroque ».

Le geste baroque

C'est-à-dire, et comme le note Jean-François Lyotard, que si « Le temps vient de ne pas s'en tenir à noter la capture et l'effacement des flux libidinaux dans un ordre dont la représentation et ses cloisons jointives et disjonctives sont, seraient le dernier mot, car cette capture et cet effacement sont le capitalisme, mais le temps vient de servir et d'encourager leur divagation errant sur toutes les surfaces et fentes immédiates crues, de corps, d'histoire, de terre, de langage ${ }^{84}$, les pratiques artistiques alternatives sont « ce temps venu ». «Temps

83 Barthes, Roland. Sade, Fourier, Loyola. Paris: Seuil, 1971. p. 10.

84 Lyotard, Jean-François. Des Dispositifs pulsionnels. Paris: Galilée, 1994. p. 23. 
venu » qui se manifeste par la mise en place de dispositifs en rupture avec les protocoles de fabrication du théâtre et les attentes de la communauté des spectateurs en se donnant, a priori, sous la forme d'un désordre esthétique. C'est-à-dire un « tournant esthétique » qui est implicitement, et également, un « tournant politique ».

Comprenons qu'il faut reconnaître et identifier à travers ces expériences théâtrales une " réponse » et une analyse aux modes de " contrôle-répression et de contrôle-stimulation ${ }^{85}$ développés par le système libéral qui, via l'industrialisation culturelle, organise conjointement la production et la réception des œuvres. D'un côté, l'organisation du goût, de l'autre une économie (organisation du coût) fondée sur « la conquête de l'audience ".

Industrie qui tend à entretenir " son propre langage avec sa syntaxe et son vocabulaire propres ${ }^{86}$, au point d'écarter la diversité des modèles.

Industrie forte d'une réussite qu'elle constate à travers un effet d'hypnose qui se traduit par un mouvement de concentration que dénoncent Adorno et Horkheimer, bien avant Habermas, où les comportements et les réactions conduisent à « faire comme tout le monde, participer à la bousculade, faire la queue... ${ }^{87}$.

Industrie qui s'observe comme la mise en place d'un « rideau idéologique ${ }^{88}$. Via ces formes qui relèvent de la communication de l'ordre établi - et « aucune pensée n'est im-

85 Foucault, Michel. Dits et Ecrits $1 \ldots$ op. cit., p. 1623.

86 Horkheimer, Max; Adorno, Theodor. La Dialectique de la raison... op. cit., p. 137.

87 Adorno, Theodor. Minima Moralia: réflexions sur la vie mutilée. Trad.: E. Kaufholz et J.-R. Ladmiral. Paris: Bibliothèque Payot, 2003. p. 160.

88 Horkheimer, Max; Adorno, Theodor. La Dialectique de la raison..., op. cit., p. 18. munisée contre les risques de la communication ${ }^{89}$ - le contrôle qui s'exerce sur l'espace sociétal : sa mémoire, son langage, l'esthétique... dispose du paysage artistique au point que le phénomène théâtral en est réduit à ne plus proposer que des spectacles où le public « vient voir sur la scène ce qu'il a dans la tête et dans le corps [...] pour se reconnaître [...] dans l'espoir de pourvoir dire à la fin : "c'est bien ça" [...] première satisfaction ${ }^{90}$.

Mais voilà qu'à travers les pratiques artistiques alternatives surgit, dans l'espace, le geste baroque.

Voilà qu'apparaît dans l'espace, alors que l'ordre libéral n'en finit pas de reconduire la communion comme le relève Jean-François Lyotard : "Aux étudiants, aux artistes, aux écrivains, aux savants, le capitalisme développé ordonnait : soyez intelligents, soyez ingénieux, vos idées sont ma marchandise d'avenir », un geste baroque lié à « l'imagination plutôt qu'au marché (et) à expérimenter sans limites ${ }^{91}$. Voilà qu'en écho au principe de dérégulation qui est le propre de l'idéologie libérale, des grupo, des collectivo s'approprient le principe de dérèglement et l'appliquent au linguistique et à l'esthétique, au risque de subir les foudres de l'arsenal judiciaire et juridique : arrestation, intimidation, procès, enfermement, expulsion ${ }^{92}$, exil...

89 Adorno, Theodor. Minima Moralia..., op. cit., p. 26.

90 Althusser, Louis. Ecrits philosophiques et politiques, tome II, Paris, Stock, 1997, p. 554

91 Jean-François Lyotard, Des Dispositifs pulsionnels...,op. cit., p. 17.

92 Voir fin 2016, l'expulsion de plusieurs grupo de l'Hospital Psiquiatrico Sao Pedro de Porto Alegre, dont, entre autres, le Teatro do Falos et Stercus dirigé par Marcelo Restori. Une partie de l'hôpital accueillait plusieurs groupes d'artistes qui avaient là leurs entrepots, leurs salles de travail, mais aussi une activité artistique auprès des internés puisque Sao Pedro est un centre actif de soins. 
Soit, et c'est l'essence même du geste baroque, un acte qui fait exister dans l'espace sociétal « un ordre sauvage ${ }^{93}$. Une réaction physique, organique, linguistique, en quelque sorte, à l'entreprise de communication, à l'arraisonnement du langage par la communication, à la disparition du langage mort d'épuisement.

Et d'ajouter qu'à travers l'apparition de ce geste baroque qui ne se réduit pas au gain d'une liberté, l'existence de cette possibilité prise et volée à l'ordre établi revient à configurer un conflit entre, d'une part le langage dramatique et son pendant la représentation, et d'autre part une « dramatisation du langage ». C'est-à-dire un questionnement sur les constituants du phénomène théâtral dès lors que l'on fait sienne la proposition de Gilles Deleuze :

[...] L'œuvre d'art n'est pas un instrument de communication. L'œuvre d'art n'a rien à faire avec la communication. L'œuvre d'art ne contient strictement pas la moindre information. En revanche, il y a une affinité fondamentale entre l'œuvre d'art et l'acte de résistance $[. . .]^{94}$. (Deleuze, 2003, p. 300).

Ce geste baroque, que d'aucuns assimileront à un « Tournant performatif », renvoie de fait, alors, à un régime “ d'inscription ${ }^{95}$ comme l'avance Jean-François Lyotard quand il pense à une rupture définitive avec la représentation classique et traditionnelle.

« Inscription » dans l'espace qui met à l'épreuve l'imagination réceptive du sujet et l'invite à vivre l'épreuve de l'imagination créa-

93 Dorléac, Laurence Bertrand. L'Ordre sauvage. Paris, Gallimard, 2004.

94 Deleuze, Gilles. Deux Régimes de fous, textes et entretiens 1975-1995. Paris: Minuit, 2003. p. 300. (Édition préparée par David Lapoujade)

95 Lyotard, Jean-François. Des Dispositifs pulsionnels...,op. cit., p. 23. trice : celle de l'artiste, la sienne aussi. Ergo, le geste baroque est le ferment de l'époché - la suspension du jugement - qui met le sujet à l'épreuve de la pensée, à l'épreuve du retour du langage, et le dispose, en définitive, à faire l'expérience du langage et de la pensée. Et si d'aventure le geste baroque n'est pas étranger à la production de significations et de sens (en cela sollicitant le logos) sous d'autres modalités esthétiques et linguistiques que celles produites par la représentation, il est surtout et essentiellement lié à l'épos : le surgissement d'intensités qui naissent à l'intersection de la rencontre de l'artiste et de son témoin.

Là, peut-être, se dessine une production partagée et retrouvée. Un « bien commun » qui construit l'idée d'un " monde en commun ». Moment où le geste baroque qui est, n'en doutons pas, un soulèvement, s'exprime également à travers le soulèvement du sujet, son geste furtif et anonyme. Instant où la conquête de l'espace réunit une pratique artistique et un sujet afin que l'espace devienne le foyer de la claire conscience où la mémoire, qui peinait à se souvenir de ce qu'est vivre la société, voit venir à elle l'idée que « la principale œuvre d'art dont il faut se soucier, la zone majeure où l'on doit appliquer des valeurs esthétiques, c'est soi-même, sa propre vie, son existence ${ }^{96}$.

Soit un premier pas, dans la rue, aux côtés du geste baroque, avec les invisibles et les délaissés, miroir des uns et des autres, les « petits " ${ }^{97}$, vers la fin d'un être sous tutelle, un être minoré... ou quand le geste baroque permet

96 Michel Foucault, Dits et écrits 2, 1976-1988, Paris, Gallimard, 2001, p. 1221.

97 Se reporter à la postface de Minima Moralia « le choix du petit " écrite par Miguel Abensour. Je cite, entre autres passages « investir contre la domination du monumental, le petit, apprendre à redécouvrir la singularité, au moment même où elle est niée " en grand " ", Theodor W. Adorno, Minima Moralia... op. cit., p. 340. 
d'envisager la disparition de cette chose que l'on appelle « le spectateur », quand l'art se fond dans la vie, sa fluidité.

Soit, pour continuer de dialoguer avec mon ami, espérer que le théâtre élargi ${ }^{98}$ (qui n'est que le déplacement du théâtre en dehors du théâtre sans porter préjudice au principe structurant de «la scène réservée ») ne soit qu'une étape (puisqu'il maintient le clivage art/spectateur, transmission de la leçon, distance et écart, etc.), avant que définitivement le grand dérèglement - comme on parle du « grand soir » - nous délivre de ce sous-sujet qu'est le spectateur tenu à distance de sa vie, tenu de regarder sa vie, figé par le regard, dans le regard, plus qu'à la vivre... Là sera la vraie révolution, le chaos ou plus simplement le retour du mouvement qu'est la subjectivation, cet état « pensant du sujet se pensant » qu'appellent de leurs vœux Deleuze et Foucault ${ }^{99}$.

Un sujet qui échapperait enfin à la « masse » et à « la compacité $^{100}$ des salles de spectacle qui sont, toujours et aussi, l'ombre des sociétés de contrôle.

«le poète est la partie réfractaire aux projets calculés »101

98 Nous dialoguons ici avec notre " jumeau " Christophe Bident qui se livre à l'analyse de BR3 d'Araujo. Christophe Bident, "Le théâtre traversé ", Théâtre/Public n 221. États de la scène actuelle 2014-2015, juillet-septembre 2016.

99 On pourrait encore citer Foucault qui explicite ce principe de subjectivation « ce qui m'étonne, c'est le fait que dans notre société l'art est devenu quelque chose qui n'est en rapport qu'avec les objets et non pas avec les individus ou avec la vie ; et aussi que l'art est un domaine spécialisé fait par des experts qui sont des artistes. Mais la vie de tout individu ne pourrait-elle pas être une œuvre d'art ? Pourquoi une lampe ou une maison sont-ils des objets et non pas notre vie ", Michel Foucault, Dits et Ecrits 2... op. cit., p. 1211.

100 Le terme de " masse » est ici emprunté à Elias Canetti. C'est à partir de la lecture de ce dernier que Miguel Abensour forge celui de "compacité ». Miguel Abensour. Miguel Abensour, De la compacité, architectures et régimes totalitaires, Paris, Sens\&Tonka 2013, p. 32-33.

101 Char, René. Recherche de la base et du sommet... op. cit., p. 33.

\section{Références}

ABENSOUR, Miguel. De la compacité, architectures et régimes totalitaires. Paris, Sens\&Tonka, 2013.

ADORNO, Theodor. Minima Moralia : réflexions sur la vie mutilée. Paris: Bibliothèque Payot, 2003.

AGAMBEN, Giorgio. La Communauté qui vient, théorie de la singularité quelconque. Paris: Seuil, 1990.

ALTHUSSER, Louis. Ecrits philosophiques et politiques, tome II, Paris, Stock, 1997.

ARENDT, Hannah. L'Humaine condition, Paris, Gallimard, 2012.

ARTAUD, Antonin. CEuvres complètes, t. V, Paris, Gallimard, 1964.

BAKHTINE, Mikhail. Le marxisme et la philosophie du langage. Trad. Marina Ya guello, Paris: Minuit, 1977.

BALANDIER, Georges. Le Désordre. éloge du mouvement. Paris: Fayard, 1988.

BARTHES, Roland. Le Bruissement de la langue. Essais critiques IV, [s.n.], Seuil, 1984.

Sade, Fourier, Loyola. Paris: Seuil, 1971.

BAUDRILLARD, Jean. D'un fragment l'autre. Paris: Albin Michel, 2011. 
BENJAMIN, Walter. Problèmes de sociologie du langage. In: CEuvres III. Paris: Gallimard, 2000.

BOUCHAIN, Patrick. In: Patrick Bouchain, pour faire avancer l'architecture, il faut de l'audace, propos recueillis par Michèle Leloup à l'Express, 13 juin 2005.

CHAR, René. Recherche de la base et du sommet. Paris: Gallimard, 1971.

CLASTRES, Pierre. Une Société contre l'État, Recherche d'anthropologie politique. Paris: Minuit, 1974.

DEBORD, Guy. La Société du spectacle. Paris: Gallimard, 1992.

DELEUZE, Gilles. Deux Régimes de fous, textes et entretiens 1975-1995. Paris: Minuit, 2003.

DELEUZE, Gilles. Pourparlers. Paris: Minuit, 1990.

DELEUZE, Gilles; GUATTARI, Felix. Mille Plateaux: Paris, Minuit, 1980.

DORLEAC, Laurence Bertrand. L'Ordre sauvage: Paris: Gallimard, 2004.

DORT, Bernard. Lecture de Brecht. Paris: Seuil, 1960.

FOUCAULT, Michel, Dits et écrits 1, 1954-1975.

Paris: Gallimard, 2001.

GALEANO, Eduardo. Les Veines ouvertes d'Amérique Latine. Paris: Plon, 1981.
GROTOWSKI, Jerzy. Le Théâtre d'aujourd'hui à la Recherche du rite. Journal France-Pologne peuples amis, n. 28-29, hiver 1968.

HEIDEGGER, Martin. Bâtir Habiter Penser. Essais et conférences. Paris: Gallimard, 1980.

HORKHEIMER, Max; ADORNO, Theodor, La Dialectique de la raison. Paris: Gallimard, 1974.

KOUNELLIS, Jannis, In « Après la scénographie », entretien dirigé par Guido Boursier, Italo Moscati et Marisa Rusconi, in Sipario, n²76, avril 1969.

LYOTARD, Jean-François. Des Dispositifs pulsionnels. Paris: Galilée, 1994.

LYOTARD, Jean-François. Textes dispersé I: esthétique et théorie de l'art. Leuven: Leuven University Press, 2012

MARCUSE, Herbert. L'Homme unidimensionnel, essai sur l'idéologie de la société industrielle avancée. Paris: Minuit, 1968.

MAURICE, Guillaume. L'archipel des délaissés. Critique, n. 757-758, Paris: Minuit, 2010, p. 542-555.

NEGRI, Antonio. Du Retour. Abécédaire biopolitique. Entretiens avec Anne Dufourmontelle. Paris: Calmann-Lévy, 2002.

NIETZSCHE, Friedrich. Le Gai savoir: aphorisme 109. Paris: Gallimard, 1982. 
RANCIERE, Jacques. Malaise dans l'esthétique. Paris: Galilée, 2004.

SANTOS, Milton. La Nature de l'espace, technique et temps, raison et émotion. Paris: L'Harmattan, 1997.

STIEGLER, Bernard. De la misère symbolique. Paris: Galilée, 2004.

Recebido: 08/05/2017 Aprovado: 10/07/2017 